\title{
Perfil Nutricional Associado ao Índice de Obesidade de Idosos do Centro de Saúde Sebastiâo Pinheiro Bastos, AAP-VR, Volta Redonda - RJ
}

\author{
Nutritional profile associated to obesity index in elderly from Sebastiáo Pinbeiro Bastos Health \\ Center, AAP-VR, Volta Redonda-RJ
}

\author{
Jair Antonio de Carvalho ${ }^{1}$ \\ André Pedrote de Carvalho ${ }^{2}$ \\ Fábio Aguiar Alves ${ }^{3}$
}

\begin{abstract}
Resumo
Trabalho autorizado pelo do Comitê de Ética do (UBM - 13/04/2006), tem como objetivo avaliar o estado nutricional e índice de obesidade de idosos voluntários do Centro de Saúde Sebastião Pinheiro Bastos, AAPVR, Volta Redonda - RJ - 2006, através do levantamento do índice de massa corporal (IMC), (OMS-1995) e da circunferência de abdome (CA), (Han e cols-1995). Foram entrevistados 1952 idosos, de ambos os sexos, maiores de 60 anos. Obteve-se os seguintes resultados para a variável (CA), risco para as doenças cardiovasculares: nas mulheres, 526 (52,97\%) apresentaram CA > $88 \mathrm{~cm}$ e 467 (47,02\%) CA < $88 \mathrm{~cm}$ (sem risco para as doenças cardiovasculares); nos homens, $460(76,79 \%) \mathrm{CA}<102 \mathrm{~cm}$ (sem risco) e $139(23,21 \%)>102 \mathrm{~cm}$ (com risco). Quanto ao IMC, foram identificados 949 idosos (59,62\%) com IMC > 24,99Kg/m2 (sobrepeso), 611 (38,37\%) com peso normal e $32(2,01 \%)$ com baixo peso. Comparando os dados obtidos com os do PNSN, (Tavares e Anjos - 1989): o baixo peso 2,01\% é inferior ao do PNSN 6,75\%; o peso normal 38,37\% é menor do que o nacional que é de 53,95\%; o sobrepeso 59,61\% é superior ao do PNSN que é de 40,30\%. O sobrepeso é muito alto, contrastando com o baixo peso e peso normal, sinalizando possível vulnerabilidade para as doenças degenerativas e suas comorbidades.
\end{abstract}

Palavras-chave: Perfil Nutricional; Índice de Obesidade; Idosos; AAP-VR

\section{Abstract}

This is a work authorized by Ethics committee (UBM-13/04/2006) and it has the objective of evaluate the nutritional state and the obesity index in volunteer elderly from Sebastião Pinheiro Bastos Health Center, AAP-VR, Volta Redonda - RJ - 2006, using the information of body mass index (BMI), (WHO-1995) and of the abdomen circumference (AC), (Hans and cols-1995). 1952 elderly were interviewed, both sexes, older than 60 years old. The following results for $(A C)$ variable, risk for cardiovascular illnesses were obtained: in women, 526 (52,97\%) presented AC>88cm and 467 (47,02\%), $A C<88 \mathrm{~cm}$ (no risk for cardiovascular illnesses); in men, 460 (76,79\%) $A C<102 \mathrm{~cm}$ (no risk) and $139(23,21 \%)>102 \mathrm{~cm}$ (risky). Regarding to BMI, 949 elderly (59,62\%) were identified with BMI > 24,99kg/m2 (overweight), 611 (38,37\%) with normal weight and 32 (2,01\%) with low weight. Comparing the obtained data with the ones from PNSN, (Tavares and Anjos - 1989): low weight 2,01\% is inferior to the one from PNSN 6,75\%; the normal weight 38,37\% is inferior to the national one, which is 53,95\%; overweight 59,61\% is superior to the PNSN, which is 40,30\%. Overweight incidence is too high if compared to the low and normal weight. It indicates a possible vulnerability for degenerative illnesses and its co morbidities.

Keywords: Nutritional Profile; Obesity Index; Elderly; AAP-VR

\footnotetext{
${ }^{1}$ Mestrando - Mestrado Profissional em Ensino em Ciências da Saúde e do Meio Ambiente - UniFOA ${ }^{2}$ Especialista - Anestesiologia - SBA

${ }^{3}$ Doutor - Mestrado Profissional em Ensino em Ciências da Saúde e do Meio Ambiente - UniFOA
} 


\section{INTRODUÇÁO}

A presente pesquisa foi desenvolvida com autorização do Comitê de Ética do Centro Universitário de Barra Mansa (UBM - 13/04/2006).

Sabe-se que o baixo-peso e o sobrepeso são fatores determinantes que comprometem a qualidade de vida de um indivíduo. Quando se trata de idosos, esses fatores têm relevância ainda maior, tendo em vista favorecer o aparecimento precoce das doenças crônico-degenerativas, o que compromete consideravelmente a vida do idoso, tanto do ponto de vista fisiológico, como do ponto de vista de autonomia e independência.

O conhecimento do perfil antropométrico de uma população pode funcionar como fator de alerta para a tomada de decisóes das autoridades de saúde, órgãos públicos, bem como para as instituiçóes assistenciais cujo foco seja o idoso, no sentido de criar programas e açóes preventivas que possam minimizar, a médio e longo prazo, as intempéries próprias da idade.

Os métodos de avaliação devem ser bem conhecidos e incluem: história nutricional, avaliação de sinais clínicos de desnutrição, peso e sua variação temporal, medidas antropométricas e determinaçôes séricas e urinárias apropriadas.

A Organização Mundial de Saúde (OMS) prevê que, em 2025, existirão 1,2 bilhóes de pessoas com mais de 60 anos, logo, o envelhecimento da população é um fenômeno de amplitude mundial. No Brasil, a situação não é diferente dos demais países latino-americanos, o país está passando por um processo de envelhecimento rápido e intenso.

Como o mundo está envelhecendo, o nosso Brasil também ficará com um número muito grande de idosos a partir do ano 2020, é de grande valia descobrir-se alguma luz que possa acompanhar esse processo degenerativo que é o envelhecimento.

A população brasileira vem envelhecendo de forma rápida desde o início da década de $60 \mathrm{e}$, de acordo com projeçôes estatísticas da (OMS), no ano de 2025, o Brasil será o sexto país do mundo com o maior número de pessoas idosas. A nutrição é um aspecto importante neste contexto pela modulação das mudanças fisiológicas relacionadas com a idade e no desenvolvimento de doenças crônicas nãotransmissíveis, como doenças cardiovasculares, diabetes, obesidade, osteoporose e alguns tipos de câncer.

A orientação nutricional é especialmente importante para os idosos devido às mudanças fisiológicas e o aparecimento de doenças relacionadas ao envelhecimento. A obesidade hoje é considerada uma doença crônica que atinge milhares de pessoas em todo mundo.

Em idosos, pequenas alteraçóes nas atividades diárias e nos hábitos alimentares podem retardar o aparecimento de muitos problemas no metabolismo e até mesmo algumas patologias, bem como, sintomas associados ao processo de envelhecimento, além de prolongar a saúde e o bem estar nos anos seguintes. Assim, um programa regular de atividade física associado a uma alimentação adequada, em termos quantitativos e qualitativos, são intervenções que melhoram ou retardam o curso de muitas doenças crônicas, oferecendo aos idosos maiores perspectivas de uma velhice feliz.

É importante uma compreensão do papel da nutrição, tanto precoce como tardia, no retardamento ou modulação do processo de envelhecimento e na promoção do estado nutricional adequado para o idoso.

Adotar uma dieta adequada é alimentar-se para uma vida saudável, retardando o envelhecimento. Ingerir alimentos ricos em nutrientes com poucas calorias faz com que o corpo fique mais saudável, com mais energia e juventude.

O presente trabalho tem como objetivo avaliar o estado nutricional e índice de obesidade de um grupo de idosos voluntários freqüentadores do Centro de Saúde Sebastião Pinheiro Bastos, da Associação dos Aposentados e Pensionistas de Volta Redonda AAP-VR, Estado do Rio de Janeiro, no ano de 2006, mediante o levantamento do Índice de Massa Corpórea (IMC) e Circunferência de Abdome (CA), identificar a incidência de baixo peso, peso normal e sobrepeso na população pesquisada.

Orientar sobre medidas que devem ser tomadas a fim de retardar a ocorrência das doenças crônicodegenerativas e minimizar o aparecimento das inabilidades funcionais, garantindo assim ao idoso maior autonomia e independência e subsidiar os profissionais da saúde e os dirigentes de instituiçóes que cuidam de idosos, na elaboração de programas e açóes que visem melhorar a qualidade de vida da população em questão, propondo medidas adequadas para que sejam adotados novos estilos de vida, com hábitos alimentares saudáveis, a fim de se obter uma melhor qualidade de vida.

Este trabalho se justifica teórica e praticamente. Em teoria, pelo fato de chamar atenção para as alteraçóes do peso e praticamente, com o direcionamento para novos estilos de vida com hábitos alimentares saudáveis, podendo com isso chegar a uma velhice com maior qualidade de vida.

\section{DESENVOLVIMENTO}

O envelhecimento é um processo gradual que tem lugar ao longo de muitas décadas. A maioria das teorias do envelhecimento relaciona-se com a replicação prejudicada 
do Ácido desoxirribonucléico (DNA) e perda da viabilidade da célula e, portanto, dos órgãos do corpo.

Os idosos são uma população mais diversificada do que qualquer outro grupo etário; os indivíduos têm capacidades e níveis de funcionamento amplamente variados. No seu todo, as pessoas idosas tendem mais que os adultos mais jovens a estar em saúde nutricional marginal e assim estão mais sujeitas ao risco de deficiência nutricional franca em situaçóes estresse ou problemas de assistência à saúde (SHILS et al, 2003). O envelhecimento populacional crescente nos últimos anos, pode trazer implicaçôes para os sistemas de saúde e para a sociedade, considerando as demandas médico-sociais, programas específicos e até mesmo recursos humanos adequados à qualidade de vida do idoso. Verifica-se atualmente que a tendência é ter um número crescente de idosos capazes de viver 20 anos mais, entretanto, podem estar funcionalmente incapacitados e com saúde precária. Nessas circunstâncias, o Brasil tem um grande desafio, pois convive com a presença crescente de doenças crônico-degenerativas (MACHADO et al, 2004).

$\mathrm{O}$ envelhecimento é um processo multifatorial que envolve uma seqüência de alteraçóes fisiológicas com perda celular e declínio dos órgãos: decréscimo da taxa de filtração glomerular (TGF) e do índice de creatinine-height (CHI), constipação, redução da tolerância à glicose e diminuição da imunidade celular podem ocorrer. A necessidade de calorias relacionadas ao metabolismo basal pode-se reduzir em até $10 \%$ na faixa de idade de 50-70 anos e em torno de 20-25\% daí em diante. Após os 70 anos de idade o peso corporal declina, a atividade física pode prevenir perdas desnecessárias na massa corporal magra. (ESCOTTSTUMP, 1999 p.29).

Um declínio na função dos órgãos, normalmente acompanha o processo de envelhecimento, especialmente nos mais idosos, ou seja, aqueles com mais de oitenta anos de idade. Pode-se razoavelmente esperar que muitas dessas alteraçôes na funçáo normal influenciem as necessidades nutricionais do indivíduo (SHILS et al, 2003).

O conhecimento das necessidades de energia por todo o ciclo da vida, durante várias condiçôes fisiológicas, como gravidez e lactação, e em vários estados de doença, é essencial para a promoção de uma saúde ótima. (MAHAN \& ESCOTT-STUMP, 2002 p.24).

A ciência da Nutrição vem se estabelecendo, nacional e internacionalmente, como instrumento básico e eficaz no alcance do bem viver; através de numerosas pesquisas sobre fatores biopsicossociais que interferem no estado nutricional; do surgimento de novas técnicas e conhecimentos sobre a composição corporal e envelhecimento e dos cuidados a serem observados na prescrição dietética e no planejamento alimentar, atendendo às necessidades nutricionais $e$ melhorando os hábitos alimentares da pessoa idosa.

O estado nutricional é, segundo a Associação Americana de Saúde Pública, "a condição de saúde de um indivíduo influenciada pelo consumo e utilização de nutrientes e identificada pela correlação de informaçôes obtidas de estudos físicos, bioquímicos, clínicos e dietéticos". Portanto, a conclusão sobre o estado nutricional pode ser obtida a partir desses estudos e correlacionam parâmetros diretos ou indiretos da situação nutricional. (AUGUSTO et al., 1993).

O estado nutricional pode afetar positivamente a expectativa de vida; o consumo nutricional e o controle das doenças aumentaram claramente o número de pessoas na população em geral que atingem uma idade que se aproxima do ciclo de vida máximo registrado. Mais recentemente, a média etária e adulta é marcada pelo aumento da longevidade (MAHAN \& ESCOTT-STUMP, 2002 p.277).

A importância da nutrição em clínica médica está sendo progressivamente reconhecida, isto em parte, é devido ao reconhecimento de que a desnutrição ocorre freqüentemente em estados prolongados da doença e pode acompanhar a lesão aguda e complicaçóes de procedimentos médicos e cirúrgicos. Muitos distúrbios genéticos metabólicos requerem dietas especiais para seu manejo. Ocorre também um aumento na compreensão do papel de fatores nutricionais em distúrbios degenerativos. (BERKOW \& FLETCHER. 1995).

A avaliação do estado nutricional é fundamental para a identificação dos pacientes sob risco nutricional. Inicialmente, deve-se buscar, na história clínica, informaçôes acerca do diagnóstico e intercorrências clínicas, que podem afetar o estado nutricional do paciente ou ser conseqüências dele. Em seguida, buscam-se evidências objetivas desse estado nutricional (antropometria, avaliação clínica e dados bioquímicos), além das intervençôes terapêuticas com interações nutricionais e, finalmente, a descrição do padrão alimentar ou o tipo de dieta que o paciente está ingerindo no momento da avaliação. (CUPPARI, 2002 p.141).

O interesse na avaliação do estado nutricional do idoso tem aumentado com a constatação de grande incidência de desnutrição neste grupo. A maioria dos casos mostra a desnutrição protéico-calórica, o que influi diretamente no sistema imunológico do idoso, tornando-o mais susceptível às doenças.

Uma das grandes preocupações da Nutrição é estabelecer, precocemente, e com maior precisão, o diagnóstico das alteraçóes do estado nutricional. Sabe-se, entretanto, que a maior parte dos casos de alteração do estado nutricional apresenta-se sob a forma subclínica, exigindo da equipe a utilização de todos os recursos disponíveis para o exame do paciente. Em circunstâncias adversas, 
o estado nutricional pode ser afetado por alteraçóes na ingestão, absorção, transporte, utilização, excreção e reserva dos nutrientes, resultando em desequilíbrio nutricional. Este, dependendo de sua intensidade e/ou duração pode comprometer o estado nutricional do organismo. Freqüentemente, verificam-se distúrbios em mais de um destes fatores e, assim, alterações que, de acordo com sua intensidade e duração, provocarão maiores ou menores sintomas e sinais clínicos (VANNUCCHI, 1996).

Vários estudos demonstram que níveis aumentados de atividade física estão associados com a redução da taxa de mortalidade e com o aumento da expectativa de vida (FRANK, 2004, p.244).

O sedentarismo, que tende a acompanhar o envelhecimento e vem sofrendo importante pressão do avanço tecnológico ocorrido nas últimas décadas, é um importante fator de risco para as doenças não transmissíveis, especialmente as afecçóes cardiovasculares, principal causa de morte nos idosos. A prática de exercício físico, além de combater o sedentarismo, contribui de maneira significativa para a manutenção da aptidão física do idoso, seja na sua vertente da saúde como nas capacidades funcionais (Alves, Mota \& Costa, 2004, p.3). O sedentarismo reduz a massa corpórea muscular, o que possibilita maior probabilidade para as quedas e fraturas em pessoas idosas. A regularidade de exercícios físicos também auxilia a fixação e manutenção do cálcio no tecido, sem contar os demais benefícios trazidos ao envelhecer com qualidade de vida (FRANK, 2004, p.148).

A obesidade e o sobrepeso são disfunçóes orgânicas em situações prevalentes nos dias de hoje e várias desordens na saúde tem sido correlacionadas com o ganho de peso em excesso. No idoso, a obesidade está associada com a hipertensão arterial, diabetes, aumento da resistência à insulina, dislipidemias, osteoartrose e muitas outras patologias, além do declínio funcional. A obesidade na população de idosos pode ser explicada pelo sedentarismo, características gerais da alimentação como excesso de lipídios e excesso de alimentos hiper-calóricos. A maior gravidade do excesso de peso no sexo feminino pode ser decorrente do maior aumento da massa adiposa e das alteraçóes hormonais específicas do sexo. (SANTOS et al., 2004, p.2).

Os "fast-foods", o sedentarismo e as disfunções orgânicas colaboram para o crescimento do número de obesos, com toda série de fatores que facilitam o aumento das células gordurosas (adipócitos) (LEIJOTO, 2000, p.247).

A obesidade, portanto, se traduz em importante condição clínica que requer uma abordagem efetiva, especialmente no que se refere à prevenção primária e secundária da coronariopatia, em que a abordagem dos fatores de risco relaciona-se com o desenho de intervençôes educacionais que possibilitem ao sujeito a adoçáo de comportamentos positivos em saúde. No entanto, a efetividade das intervençóes voltadas para o controle desses fatores pressupóe, num primeiro momento, o conhecimento de como eles se distribuem na população a qual se destinam tais intervençôes (Colombo et al, 2003). Recentes estudos sugerem que os genes fornecem susceptibilidade para a obesidade, mas não são a sua causa efetiva. Isso significa que embora os genes pareçam aumentar a vulnerabilidade à obesidade, outros determinantes devem estar presentes para a ocorrência da obesidade. O maior fator é o ambiental (MAHAN \& ESCOTT-STUMP, 2002 p.478).

Antropometria é a medida das dimensôes corpóreas; as medidas antropométricas mais empregadas na avaliação do estado nutricional são: peso, altura, circunferências (braço e cintura) comprimento do braço e pregas cutâneas (tríceps, bíceps, subescapular, supra-ilíaca). Através da combinação destas medidas, pode-se calcular as relaçóes peso/altura2 e a circunferência muscular do braço e o índice de gordura do braço (Vannucchi, 1996). Na avaliação antopométrica, como em qualquer processo de avaliação nutricional individual ou coletivo, torna-se necessário que o avaliador tenha a consciência de que não está lidando com um objeto, e sim com um indivíduo, com toda a sua sensibilidade, história de vida e fragilidade, respeitando a individualidade de cada um (FRANK, 2004, p.27).

O indicador antropométrico é essencial na avaliação nutricional geriátrica, entretanto, algumas alterações que ocorrem com o envelhecimento podem comprometer a determinação de um diagnóstico antropométrico acurado e preciso, caso, cuidados específicos náo sejam tomados no sentido de neutralizar ou amenizar o efeito dessas alteraçóes sobre a avaliação (SAMPAIO, 2004).

Aantropometriatem semostradoimportanteindicador do estado nutricional. Além de fornecer informaçôes das medidas físicas e de composição corporal, é método não invasivo e de fácil e rápida execução (MENEZES, 2005).

Os estudos antropométricos e dietéticos, atualmente alcançam definitiva importância por tratarem de aspectos intimamente relacionados com a qualidade de vida pretendida para esta parcela da sociedade em todo mundo. Não há dúvida de que os indicadores antropométricos contribuem na avaliação do estado nutricional, em diferentes momentos fisiológicos e fisiopatológicos, de diversos grupos etários. (FRANK, 2004, p.173).

As medidas antropométricas representadas pelo Índice de Massa Corpórea (IMC) e Circunferência de abdome (CA) representam uma maneira racional e eficiente de se presumir o volume e as distorçóes de gordura, devendo assim, ser utilizada na prática clínica cotidiana. 
Nas últimas décadas, em função de uma maior preocupação com a obesidade endêmica no mundo, IMC tem sido retomado na avaliaçáo do estado nutricional. (TEIXEIRA NETO, 2003 p.142).

O IMC é determinado a partir das variáveis peso e estatura, que consiste na medida do peso corporal $(\mathrm{Kg})$, dividido pela estatura ( $\mathrm{m}$ ) elevada ao quadrado, logo, $\mathrm{P} /$ $\mathrm{E}^{2}$. O padrão de referência utilizado, baseia-se nos dados do Comitê de Experts da OMS, em 1995, sendo: IMC menor que $18,50 \mathrm{~kg} / \mathrm{m}^{2}$ para subnutrição ou baixo peso, IMC entre $18,50 \mathrm{~kg} / \mathrm{m}^{2}$ e $24,99 \mathrm{~kg} / \mathrm{m}^{2}$ para normalidade e IMC maior que $24,99 \mathrm{~kg} / \mathrm{m}^{2}$ para sobrepeso ou obesidade (TEIXEIRA NETO, 2003 p.142).

Medições do peso corporal e da altura são feitas com facilidade e são de grande utilidade na avaliação do crescimento e do estado nutricional (SHILS et al, 2003).

A aferição do peso, embora seja simples, de fácil aplicaçáo e seja ampla a disponibilidade de equipamentos, é negligenciada com freqüência na admissão do paciente. O peso, que representa o somatório de todos os compartimentos corporais, é de limitado valor, por si só, na avaliação nutricional do adulto, contudo, quando comparado à altura, peso habitual ou peso ideal, fornece índices importantes, na avaliação nutricional, que podem auxiliar na identificação tanto da desnutrição quanto da obesidade. (TEIXEIRA NETO, 2003 p.141).

$\mathrm{Na}$ meia idade (50 a 65 anos), o maior problema nutricional é o sobrepeso. Valores elevados de IMC estão associados a várias doenças crônicas. Acima de 80 anos, magreza e perda de massa magra são os maiores problemas. Baixos valores de IMC estáo associados a tuberculose, distúrbios pulmonares obstrutivos, câncer de pulmáo e estômago. Os estudos longitudinais têm demonstrado que tanto sobrepeso quanto magreza consistem em risco de morte, mas no idoso a magreza consiste em risco maior. O comportamento do IMC através do avanço da idade é semelhante ao que acontece com o peso, havendo declínio por volta dos 70 a 75 anos (FRANK, 2004, p.18).

A altura é uma medida antropométrica linear de simples aplicação e pouco utilizada na prática clínica, apesar da disponibilidade de equipamentos para sua aferição. Embora seja fundamental na avaliação nutricional da criança, a altura é de pouca valia na avaliaçáo nutricional de adultos, no entanto, é utilizada em importantes índices de avaliação nutricional, como IMC, e o índice de creatinina por altura e em equaçóes estimativas do gasto energético (TEIXEIRA NETO, 2003 p.142).

A doença Arterial Coronariana (DAC) é responsável pela maior taxa de morbidade e mortalidade no mundo ocidental. Isso corre devido a uma série de fatores, dentre eles a maior longevidade dos indivíduos, o que, por conseguinte, daria maior tempo para a doença se manifestar (FRANK, 2004, p.82). A Circunferência de Abdome é medida em centímetros, no meio da distância entre a crista ilíaca e o rebordo costal inferior. Por ser o índice antropométrico mais representativo da gordura intra-abdominal e de aferição mais simples, é a medida recomendada (BRANDĀO, 2005). Os valores de Circunferência de Abdome que correspondem ao aumento de risco variam de acordo com a idade e o sexo. Segundo Han e cols., a partir de $102 \mathrm{~cm}$ em homens e 88 $\mathrm{cm}$ nas mulheres, há um risco substancialmente aumentado de complicaçôes metabólicas associadas à obesidade (CABRERA, 2001).

Em idosos, no entanto, torna-se mais complexa a análise do estado nutricional em virtude da maior heterogeneidade entre os indivíduos e pelo fato de seu valor preditivo estar atrelado a um conjunto de fatores não apenas relacionados às mudanças biológicas da idade, doenças e mudanças seculares, como também às práticas ao longo da vida (fumo, dieta, atividade física) e aos fatores sócioeconômicos (TAVARES \& ANJOS, 1999, p.2).

\section{MATERIAIS E MÉTODOS}

Pesquisa transversal desenvolvida no Centro de Saúde Sebastião Pinheiro Bastos da Associação dos Aposentados e Pensionistas de Volta Redonda, RJ (AAP-VR). Onde foram entrevistados 1592 idosos voluntários, no ano de 2006, de ambos os sexos, maiores de 60 anos, freqüentadores da Instituição. Os voluntários foram medidos e pesados, bem como aferida a circunferência de abdome, na sala de espera do referido centro de saúde, pelo nutricionista responsável por esta pesquisa. A pesagem e a altura dos voluntários foi aferida em balança com travessão e pesos móveis e estadiômetro, de marca "welmy", onde foi solicitado aos voluntários que tirassem os calçados. A aferição da circunferência de abdome foi feita com fita métrica convencional.

A Associação dos Aposentados e Pensionistas de Volta Redonda é uma entidade de utilidade pública e filantrópica, que tem como objetivo prestar assistência aos seus associados, defendendo seus direitos e interesses. Surgiu da uniấo e da organização da classe dos aposentados e pensionistas e hoje é a maior Associação de Aposentados da América Latina, prestando assistência médica, odontológica, social e funerária aos associados e aos seus dependentes. Criada em 1973, inicialmente atuando com muita dificuldade, hoje a AAP-VR é grandiosa porque nasceu com um objetivo grandioso: o de lutar pela dignidade daqueles que tanto deram de si para construir o presente e o futuro do país.

Inicialmente ocupava sedes provisórias e hoje possui um sólido patrimônio, com sede próprias, modernas e 
acessíveis, que oferecem conforto e segurança aos seus funcionários e associados. Conta hoje com mais de 45 mil associados.

A amostra representa $17,68 \%$ da população atendida no referido Centro, estimada em 9.000 participantes, no período de desenvolvimento da coleta de dados.

Os dados foram coletados de 16 de Janeiro a 16 de Março de 2006, nos períodos matutino e vespertino em semanas alternadas, através de entrevista individual, conforme formulário a seguir apresentado.

Código: $\quad$ FICHA DE AVALIAÇ̃̃o DE IDOSOS
Idade:__ Peso:__ Altura:__ Circunferência de Abdome:__ Atividade Física: S ( ) N ( )
Sexo:M ( ) F ( ) Fumante:S ( ) N ( )Álcool:S ( ) N ( ) Tem animal de
companhia:S ( ) N ( )
Doenças:

Peso Corporal: a pesagem dos idosos foi realizada em balança de travessão com pesos móveis de marca WELMY. Para aferir o peso corporal, o indivíduo ficou na plataforma da balança, com o peso do corpo igualmente distribuído entre os pés, conforme o método descrito por (HEYWARD \& STOLARCZYK, 2000).

Estatura: foi utilizado estadiômetro com uma haste móvel para medir a altura em pé. O idoso descalço, foi posicionado em uma superfície plana, em ângulo reto com a haste vertical ou prancha de estadiômetro. Os pés estavam juntos, formando um ângulo de $60^{\circ}$, com a cabeça, escápula e glúteos tocando a prancha vertical, cabeça ereta e olhos fixos na frente. Conforme o método descrito por (HEYWARD \& STOLARCZYK, 2000).

Índice de Massa Corporal (IMC): Para calcular o IMC, o peso foi aferido em quilogramas $(\mathrm{kg})$ e a altura em metros $(\mathrm{m})$. Os limites de corte para sobrepeso e emagrecimento foram propostos pelo Comitê de Experts da OMS, em 1995. Sendo: IMC menor que $18,50 \mathrm{~kg} / \mathrm{m}^{2}$, emagrecimento; de $18,50 \mathrm{~kg} / \mathrm{m}^{2}$ a $24,99 \mathrm{~kg} / \mathrm{m}^{2}$, variação normal e maior que $24,99 \mathrm{~kg} / \mathrm{m}^{2}$ sobrepeso (TEIXEIRA NETO, 2003 p.142).

Circunferência de Abdome: Foi aferida com fita métrica, em centímetros conforme estabelecido na I Diretriz Brasileira de Diagnóstico e Tratamento da Síndrome Metabólica.

\section{RESULTADOS}

A amostra consta de 1592 idosos entrevistados e estes foram "distribuídos" segundo as variáveis:

Sexo: Dos entrevistados, $62,37 \%$ eram do sexo feminino e $37,62 \%$ do sexo masculino.

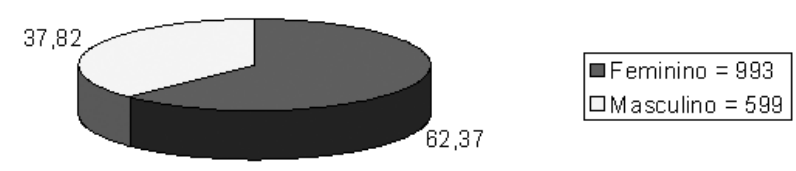

Gráfico 1 - Distribuição por Sexo, 2006.

Idade: A idade média de 65 anos, destes $57 \%$ era com idade entre 60 e 69 anos, 33\% com idade entre 70 e 79 anos e $10 \%$ maiores de 79 anos.
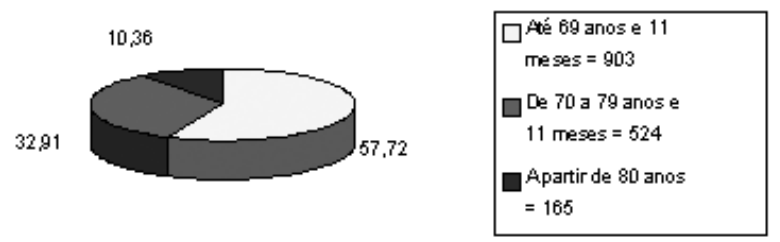

Gráfico 2 - Distribuição por Idade, 2006.

Circunferência de Abdome: Para esta variável obteve-se os seguintes valores para o sexo feminino:

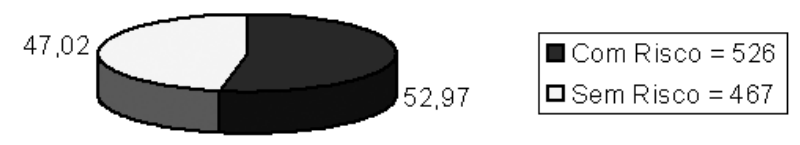

Gráfico 3 - Risco Cardiovascular - Feminino, 2006.

O índice obtido no presente trabalho para a variável CA em mulheres $(52,97 \%)$, com risco para doenças cardiovasculares, é um percentual alto, especialmente quando comparado com os dados de $38,55 \%$ que Diasda-Costa, 2006 obteve em pesquisa feita na cidade de Pelotas, RS, entre os anos de 1999 e 2000. Em trabalho desenvolvido por Colombo et al, com pacientes idosos enfartados, atendidos nun serviço de prevenção secundária, registrou uma prevalência de $64 \%$ dos casos com risco para doenças cardiovasculares, ou seja, com índices maiores que os desejados.

Para o sexo masculino, obteve-se os seguintes valores:

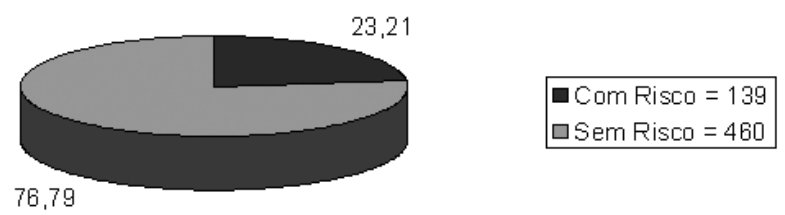

Gráfico 4 - Risco Cardiovascular - Masculino, 2006.

O índice obtido no presente trabalho para a variável CA em homens (23,20\%), com risco para doenças cardiovasculares, é um índice satisfatório, quando comparado aos dados de Dias-da-Costa, 2006 em pesquisa feita na cidade de Pelotas - RS, entre os anos de 1999 e 2000, que é de 18,49\%.

Comparando os resultados obtidos para CA, nos 
dois trabalhos, observa-se que as idosas apresentam maior percentual de risco para as doenças cardiovasculares, quando comparados aos do sexo masculino.

IMC: Para o cálculo do IMC foi tomada como referência a classificação recomendada pela OMS (WHO, 1995), que preconiza para baixo peso IMC menor que $18,5 \mathrm{~kg} / \mathrm{m}^{2}$, peso normal IMC > de $18,49 \mathrm{~kg} / \mathrm{m}^{2}$ e menor de $24,99 \mathrm{~kg} / \mathrm{m}^{2}$ e sobrepeso IMC > de $24,99 \mathrm{~kg} / \mathrm{m}^{2}$. (TEIXEIRA NETO, 2003 p.142).

Segundo o IMC obteve-se: 32 idosos (2,01\%) com IMC menor de $18,50 \mathrm{~kg} / \mathrm{m}^{2} ; 611(38,37 \%)$ com IMC maior de $18,50 \mathrm{~kg} / \mathrm{m}^{2}$ e menor de $25,00 \mathrm{~kg} / \mathrm{m}^{2}$ e 949 $(59,61 \%)$ com IMC maior que $25,00 \mathrm{~kg} / \mathrm{m}^{2}$.
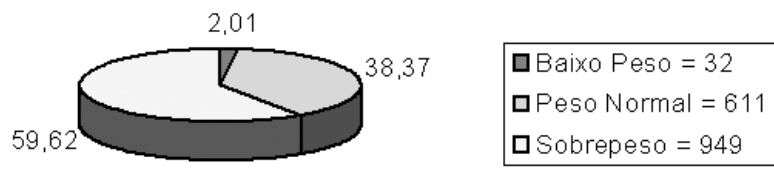

Gráfico 5 - Classificação por IMC, 2006.

\section{DISCUSSÃO}

A maioria dos idosos classifica-se na faixa de sobrepeso, IMC maior que 25,00 kg/m2 (59,62\%), é importante observar que é um índice muito alto para sobrepeso.

Entre os idosos que apresentam sobrepeso, foi registrado 586 casos $(61,74 \%)$, classificados como sobrepeso grau I (IMC maior que $24,99 \mathrm{~kg} / \mathrm{m}^{2}$ e menor que $30,00 \mathrm{~kg}$ / $\mathrm{m}^{2}$ ); 344 casos (36,24\%), classificados como sobrepeso grau II (IMC maior que $29,99 \mathrm{~kg} / \mathrm{m}^{2}$ e menor que $39,99 \mathrm{~kg} / \mathrm{m}^{2}$ ) e 19 casos $(2,00 \%)$, classificados como sobrepeso grau III (IMC maior que $39,99 \mathrm{~kg} / \mathrm{m}^{2}$ ).

$\mathrm{O}$ índice para baixo peso $(2,01 \%)$, caracteriza-se como um bom resultado, porém é importante observar.

Entre os idosos que apresentam baixo peso, foi registrado 20 casos $(62,50 \%)$, classificados como baixo peso leve (IMC maior que $16,99 \mathrm{~kg} / \mathrm{m}^{2}$ e menor que 18,50 $\left.\mathrm{kg} / \mathrm{m}^{2}\right) ; 7$ casos $(21,87 \%)$, classificados como baixo peso moderado (IMC maior que $15,99 \mathrm{~kg} / \mathrm{m} 2$ e menor que $\left.17,00 \mathrm{~kg} / \mathrm{m}^{2}\right)$ e 5 casos $(15,62 \%)$, classificados como baixo peso grave (IMC menor que $16,00 \mathrm{~kg} / \mathrm{m}^{2}$ ).

Tavares e Anjos (1999), em Pesquisa Nacional Sobre Saúde e Nutrição (PNSN), desenvolvida entre Junho e Setembro de 1989, com o objetivo de aferir o estado nutricional da população brasileira, obteve os seguintes resultados: $6,75 \%$ para baixo peso, $52,95 \%$ para peso normal e $40,30 \%$ para sobrepeso.

$\mathrm{O}$ índice para peso normal $(38,37 \%)$ apresenta-se muito baixo, uma vez que o índice nacional (PNSN) é de $52,95 \%$. Entre os idosos que apresentam peso normal, foi registrado 611 casos $(38,37 \%)$, com IMC maior que 18,49 $\mathrm{kg} / \mathrm{m}^{2}$ e menor que $25,00 \mathrm{~kg} / \mathrm{m}^{2}$.

É interessante observar que para o fator sobrepeso os valores são bem diferentes $(59,61 \%$ e $40,30 \%)$, enquanto que para baixo peso, há uma diferença considerável (2,01\% e 8,75\%). Para o fator normalidade, há também uma diferença considerável $(38,37 \%$ e 52,95\%), desfavorável para a população da amostra em questão.

\section{CONCLUSÃo}

No Brasil, vários trabalhos foram realizados, envolvendo população idosa, institucionalizada ou não. No entanto, ainda são necessários mais estudos antropométricos com esse segmento da sociedade.

Após avaliar mil quinhentos e noventa e dois (1592) idosos voluntários, entre estes, sendo novecentos e noventa e três (993) do sexo feminino e quinhentos e noventa e nove (599) do sexo masculino, foi obtido um resultado apresentando alta incidência de sobrepeso, fator este que predispóe à ocorrência das doenças degenerativas, que acomete a população com idade de quarenta (40) anos ou mais.

Além da alta incidência de sobrepeso, foi registrada para a variável Circunferência de Abdome (CA) uma ocorrência muito elevada de valores superiores ao desejado, para os idosos do sexo feminino, logo, com risco para as Doenças Cardiovasculares.

Avaliando os dados obtidos na pesquisa, conclui-se que o índice de sobrepeso apresenta-se muito alto, contrastandose com o nível de baixo peso. O percentual para eutróficos apresenta-se muito baixo. Tais resultados permitem detectar a grande vulnerabilidade da população em questão, para as doenças degenerativas e suas co-morbidades.

Portanto, medidas preventivas devem ser tomadas a fim de evitar que o quadro atual do perfil antropométrico da população se torne ainda mais comprometedor.

Medidas terapêuticas, dietéticas e educacionais devem ser aplicadas, a fim de minimizar, se não erradicar o problema do sobrepeso, garantindo assim uma melhor qualidade de vida, com mais saúde, agregando mais anos de vida, e o que é mais importante, uma longevidade mais feliz.

\section{REFERÊNCIAS}

ALVES, R.V.; MOTA, J.; COSTA, M.C. Aptidão física relacionada à saúde de idosos: influência da hidroginástica. Rev. Brasileira Medicina e Esporte. Vol.10, n.1, p.3, Rio 
de Janeiro: jan./fev. 2004.

AUGUSTO, A.L.P.; ALVES, D.C.; MANNARINO, I.C.; GERUDE, M. Terapia Nutricional. São Paulo: Atheneu, 1993.

BERKOW, R., FLETCHER, A.J. Manual Merck de medicina: diagnóstico e tratamento. São Paulo: Roca, 1995.

BRANDĀO, A.P.I. Diretriz Brasileira de Diagnóstico e Tratamento da Síndrome Metabólica. Rev. Arquivos Brasileiros de Cardiologia. Vol. 84, n.7, Sáo Paulo: 2005.

CABRERA, M.A.S.; JACOB, W. Obesidade em idosos: Prevalência, Distribuição e Associação com Hábitos e CoMorbidades. Rev. Arquivos Brasileiros de Endocrinologia e Metabolismo. São Paulo: Vol. 45 n.5, 2001.

COLOMBO, R.C.R., et al. Caracterização da Obesidade em Pacientes com Infarto do Miocárdio. Rev. LatinoAm Enfermagem. Vol. 11, n.4, Ribeirão Preto: July/Aug. 2003.

CUPPARI, L. Nutrição Clínica no Adulto. São Paulo: Manole, 2002.

DIAS-DA-COSTA, J.S., et al. Níveis de Intervenção para obesidade Abdominal: Prevalência e Fatores Associados. Cad. Saúde Pública. V.22, n.6 Rio de Janeiro: jun. 2006.

ESCOTT-STUMP, S. Nutriçáo relacionada ao diagnóstico e tratamento. São Paulo: Manole, 1999.

FRANK, A.A.; SOARES, E.A. Nutrição no Envelhecer. São Paulo: Atheneu, 2004.

HAN, T.S. et al. Obesidade em Idosos: Prevalência, Distribuição e Associação Com Hábitos e Co-Morbidades. Arquivos Brasileiros de Endocrinologia e Metabologia. Acessado em www.scielo.br/scielo em 15/03/06.

HEYWARD, H.V. \& STOLARCZYK M.L. Avaliação da Composição Corporal Aplicada. São Paulo. Manole, 2000 .

LEIJOTO, C.M. Sua Saúde no Novo Milênio. São Paulo: Tecnopress, 2000.

MACHADO, J.S.; SOUZA, V.V.; VIDAL, A.T.; RAMOS, F.R.M.; FRANK, A.A. Perfil nutricional de idosos freqüentadores da Associação Recreativa de Copacabana. Rio de Janeiro: Rev. Nutriçáo Brasil. Ano 3, n.2, Rio de Janeiro: Março/Abril 2004.

MAHAN, L.K.; ESCOTT-STUMP, S. Krause Alimentos, Nutriçáo \& Dietoterapia. São Paulo: Roca, 2002.

MENEZES, T.N., MARUCCI, M.F.N. Antropometria de idosos residentes em instituiçôes geriátricas. Rev. Saúde Pública. Vol. 39, n.2, São Paulo: Abril 2005.

SAMPAIO, L.R. Avaliação Nutricional e Envelhecimento. Revista de Nutriçáo. Vol. 17, n.4, Campinas: Outubro/ Dezembro 2004.

SANTOS, C.L. et al. Indicadores do estado nutricional de idosos institucionalizados. Rev. Nutriçáo Brasil. Vol. 5, n.8, Campinas: maio/junho. 2004.

SHILS, M.E., et al. Tratado de Nutriçáo Moderna na Saúde e na Doença. 9.ed. São Paulo: Manole, 2003.

TAVARES, E.L.; ANJOS, L.A. Perfil Antropométrico da população idosa brasileira: resultados da Pesquisa Nacional sobre Saúde e Nutrição. Caderno de Saúde Pública. Vol. 15, n.4, São Paulo: out./dez.1999.

TEIXEIRA NETO, F. Nutrição Clínica. Rio de Janeiro. Guanabara Koogan, 2003.

VANNUCCHI, H.; UNAMUNO, M.R.D.L.; MARCHINI, J.S. Avaliação do estado nutricional. Rev Medicina Ribeirão Preto. N. 29, p. 5 -18, Rio de Janeiro: jan/mar. 1996.
Endereço para Correspondência:

Jair Antonio de Carvalho carvalho@superonda.com.br Mestrado Profissional em Ensino em Ciências da Saúde e do Meio Ambiente

Centro Universitário de Volta Redonda Campus Três Poços Av. Paulo Erlei Alves Abrantes, no 1325 , Três Poços - Volta Redonda / RJ CEP: 27240-560 